Rev. salud pública. 12 sup (2): 86-90, 2010

Poster Presentation

\title{
Clinical aspects of mycobacteria
}

\author{
Infant tuberculous meningitis
}

\author{
Maira Juliana Chamorro, Magnolia Arango \\ Departamento de Pediatría, Universidad Nacional de Colombia.
}

Summary: Male of 2 years and 8 months of age, from Ortega (Tolima), Colombia, with 7 days of fever; multiple, generalized, tonic-clonic convulsions; and motor focalization.History: Home birth, without vaccination; resided during the past 12 months with an uncle with TB, under treatment during the past 6 months.Clinical examination: Sick, feverish, dehydrated, without a BCG scar; sleepy, ataxic with center-right facial paralysis, without meningeal signs. Radiography of thorax: left parahiliary calcified lesions. Treatment was indicated with cefepime + vancomycin. Bacilloscope smears of gastric juice was negative and tuberculine of $9 \times 12 \mathrm{~mm}$. Anti-TB treatment was initiated on july 22 . A neurological deterioration was presented with endocraneal hypertension and active hydrocefalis treated with ventriculostomy. Consistent fever and convulsive episodes were observed despite antibiotics, which were suspended due to negative blood and CSF cultures.On August 16, 2008, a positive RCP was reported in CSF for M. tuberculosis. The $24^{\text {th }}$ day of anti-TB therapy revealed hepatic insufficiency; it was therefore necessary to reduce the dosage of anti-TB treatment. The patient's condition remains unchanged, and the neurological compromise is very significant; the possibility of limiting therapeutic efforts has been discussed with the parents. This demonstrates the consequences of a late and inappropriate diagnosis in spite of epidemiological data and clinical history.

\section{Tuberculosis pneumonia in a child}

Pablo Vasquez and Magnolia Arango

Universidad Nacional de Colombia. HOMI. Bogota, Colombia.

This is a case of a one year old female patient, born in Coyaima (Tolima), with fever, anorexia, frequent crying, rapid weight loss and coughing for the past 3 months prior to her first consult. First a diagnosis o malnutrition was made. With no improvement she was suspected of having tuberculosis that was confirmed with a positive gastric aspirate. Given that there was no improvement after 3 weeks of treatment, she was transfer to our care. Born to a 26 year old mother, $1^{\text {st }}$ pregnancy, healthy newborn. Complete vaccination schedule for age including BCG. Her mother had fever, chills, anorexia, persistent cough and weight lost for 7 months and was diagnosed with pulmonary tuberculosis. At the time of diagnosis, our patient and her family were tested and were all negative. Physical examination revealed a wasted patient with respiratory distress, no BCG scar. Rattles on both lung fields. A chest $\mathrm{X}$ ray showed left lower and right upper lobe opacities, with areas of focal necrosis. HIV was 
negative. Spinal fluid was normal but gastric aspirates were still positive for tuberculosis. Treatment was readjusted at a higher therapeutic dose. Some samples were send to the Mycobacterial Laboratory (Universidad Nacional de Colombia), were they found a positive PCR in the spinal fluid and gastric aspirates. Cultures of were negative. The patient has improved since the treatment was readjusted.

We bring this case to discuss the implications of postponing the diagnosis that if taken in consideration the epidemiological and clinical data, she could have received a more appropriated, timely, comprehensive treatment.

\section{Primary prostatic tuberculosis: a very rare form of presentation of the tuberculosis infection}

Eliana Lopez Baron, Diego Gomez-Arbelaez, Julio Alexander Diaz-Perez, Julio Cesar Mantilla Hernandez

Departamento de Patología, Universidad Industrial de Santander, Bucaramanga, Colombia.

INTRODUCTION: The primary prostatic tuberculosis is a very rare form of presentation of the tuberculous infection, which is generally caused by the M. tuberculosis, and which has shown an increase in incidence and prevalence, due to an increase of immunocompromised patients and the pandemic of the Syndrome of Acquired Immune Deficiency (AIDS). OBJECTIVE: To describe a case of primary prostatic tuberculosis attended at the Hospital Universitario de Santander, Colombia, and to perform a discussion about this topic. CLINIC CASE: 65 years old man who consulted with symptoms of frequency, dysuria and hesitancy, and lost of weight of $10 \mathrm{Kg}$ in the last 6 months, without pulmonary symptoms and negative ELISA test for HIV. In the physical exam was evidenced the presence of a high volume, irregular and hard prostatic gland. That is why was performed a prostatic Doppler ecography that show a prostatic volume of $39 \mathrm{~cm} 3$, without indicating characteristics of malignity. The puncture biopsy of the prostatic gland shows multiple granulomas and the $\mathrm{ZN}$ staining was positive for micobacterias. With the earlier findings was performed the diagnostic of primary prostatic tuberculosis, which was treated and now the patient is asymptomatic with no evidence of active tuberculosis. CONCLUSIONS: Primary prostatic tuberculosis without history or evidence of commitment of the immune system, is a very rare condition, despite this knowledge is particularly important because of the progressive increase of its presentation and the possibility of a curative treatment to affected patients.

Key Words: Primary prostatic tuberculosis, urogenytal tuberculosis, granulomatous prostatitis, tuberculosis, prostate, (Source: $\mathrm{MeSH}$ ). 


\title{
Morphological patterns of tuberculosis: series of fatal cases at Hospital Universitario of Santander between January 2003 and December 2006
}

\author{
Julio Cesar Mantilla Hernández, José Arnulfo Pérez Carrillo y \\ Nelson Cárdenas Durán
}

Departamento de Patología Universidad Industrial de Santander.

Twenty five years ago, Tuberculosis (TB) was a well-controlled illness, with very well-defined clinical and paraclinical diagnosis and handling parameters. The outbreak of Human Immunodeficiency Virus (HIV) infection, together with raised malnutrition levels, has resulted in a changed clinical manifestation of TB. OBJECTIVE: To conduct fatal TB necropsy case series along last four years. MATERIALS AND METHODS: 738 necropsy protocols conducted at the morgue of Hospital Universitario of Santander by the Pathology Department at the Universidad Industrial of Santander between January 1st 2003 and December 31st 2006 were reviewed of which 17 protocols corresponded to fatal TB cases. Additionally, MedLine Literature was analyzed including a comparison of result to series similar to that of this study. RESULTS: 17 fatal TB cases are included, presented in four morphological patterns: seven cases $(41,2 \%)$ of Disseminated TB, four cases $(23,5 \%)$ of pulmonary miliary TB, four cases $(23,5 \%)$ isolated organ TB, and two cases $(11,7 \%)$ of cavitary pulmonary TB. CONCLUSION: TB changed its most requent cavitary pulmonary TB manifestation into disseminated military orm affecting predominantly lung, lymph node, spleen, liver, kidney and drenal gland of HIV-infected patients or extreme malnutrition cases. Increased central nervous system compromise is evident not only as eningitis with outstanding vasculitis but also as abscess and Tuberculoma. The series also shows slight compromise of organs such as prostate, estes, uterus and small intestine.

Key Words: TB necropsy, cavitary, Disseminated, pulmonary TB.

\section{Congenital tuberculosis associated with maternal disseminated miliary tuberculosis}

Julio Cesar Mantilla Hernandez, Luís Miguel Sosa, Luz Libia Cala y Nelson Cardenas

Departamento de Patología, Universidad Industrial de Santander, Bucaramanga, Colombia.

Untreated tuberculosis during pregnancy presents a serious risk for transmission of disease to the newborn and can result in adverse perinatal and obstetrical outcomes. Tuberculosis during pregnancy and congenital tuberculosis are infrequent conditions and are difficult to diagnose due the non-specificity of the symptoms. A case report is presented of a woman who had no children previously with disseminated miliary tuberculosis. Tuberculosis symptoms appeared immediately after birth of the first child, with a clinical diagnosis on the second month after childbirth, whereupon the patient died. The son, a premature infant, showed disease symptoms from the first day, with primary pulmonary complex and persistent 
atelectasis due to bronchial obstruction. The obstruction was due to thoracic lymphadenitis and coinfection with cytomegalovirus. The infant received standard treatment and his condition improved.

Key Words: Congenital tuberculosis, miliary tuberculosis, infant.

\section{Mesotherapy and Mycobacteria}

Dora Orjuela ${ }^{1}$, Gloria Puerto ${ }^{1}$, Graciela Mejía ${ }^{1}$, Claudia Castro $^{1}$, Maria Consuelo Garzón $^{1}$, Luz Mary García ${ }^{1}$, Elkin Hernández ${ }^{2}$, Wellman Ribon ${ }^{1}$, Gerzaín Rodriguez ${ }^{2}$

1 Grupo de Micobacterias, Instituto Nacional de Salud, Bogotá DC, Colombia.

2 Grupo de Biología Molecular de las Micobacterias, Facultad de Medicina, Universidad de La Sabana, Chía, Cundinamarca, Colombia.

INTRODUCTION: Intradermic injection of compounds with therapeutic aims, cosmetics principally, have caused abscesses by Nontuberculous mycobacteria (NTM). It is known a report from Colombian about cutaneous tuberculosis for this procedure. OBJECTIVE: Review concepts on the more than 400 cases of skin lesions by NTM obtained at INS. Report 6 adults which developed cutaneous tuberculosis by mesotherapy. METHODS: In gluteal and abdominal intradermic injection of not specified material, five women and one man developed papules, subcutaneous nodules and formation of draining sinuses. Cultures of biopsies and secretions were performed for phenotypical identification of mycobacteria. Genotypic identification was conducted using PCR-restriction analysis "PRA" and spoligotyping. RESULTS: In five of six patients was demonstrated $M$. tuberculosis by phenotypic and genotypic identification tests. Five cured with antimycobacterial therapy and one spontaneously after resection of lesions. Satellite adenopathies and recurrences were not documented. None had TB elsewhere. CONCLUSION: Mesotherapy can produce cutaneous tuberculosis. Source of origin of bacillus was not dertemined. The presence of the bacillar DNA in tissue sections included in paraffin and growth in culture of lesions discard laboratory contamination. Any injury at the injection site after mesotherapy procedure should suggest mycobacterial infection including search of M. tuberculosis. Health authorities should pay attention to mycobacterial infections caused by mesotherapy that are frequently in all country.

Key Words: Cutaneous tuberculosis, human mycobacteriosis, mesotherapy.

Mycobacteria in a HIV-1 infected population from São Paulo State, southeastern Brazil in the post HAART era

Heloisa da Silveira Paro Pedro ${ }^{1,4}$, Maria Izabel Ferreira Pereira ${ }^{1}$, Maria do Rosário Assad Goloni ${ }^{1}$, Fernanda Carina Pires ${ }^{1}$, Rosângela Siqueira Oliveira ${ }^{2}$,

Maria Aparecida Batista da Rocha ${ }^{3}$, Luciana Moran Conceição ${ }^{4}$, Valéria Daltibari

Fraga $^{4}$, Juliana Camargo Fenley ${ }^{4}$, Talita Cordeschi ${ }^{4}$, Ricardo Luiz Dantas Machado ${ }^{4}$, Célia Franco ${ }^{5}$, Andréa Regina Baptista Rossit ${ }^{4}$ 


\begin{abstract}
1 Adolfo Lutz Institute - Regional Laboratory from São José do Rio Preto.
2 Adolfo Lutz Institute - Central Laboratory from São Paulo.

3 DST/AIDS Specialized Attendance Service - São José do Rio Preto.

4 Center for Microorganisms Investigation, Department of Dermatological, Parasitical and

Infectious Diseases from Faculty of Medicine from São José do Rio Preto and

5 Infectious and Parasitical Diseases Service from Hospital de Base - Faculty of Medicine from São José do Rio Preto Foundation, São Paulo State, Brazil.
\end{abstract}

São José do Rio Preto city (SJRP), Northwestern São Paulo State, Brazil, is considered "priority" by the National Programs of Tuberculosis and AIDS Control. Our purpose was to retrospectively evaluate Mycobacterium sp. isolated from HIV-infected patients attending the HIV/TB reference health care units from SJRP and region, as well to describe their clinical and socio-demographic aspects. One hundred and ninety-eigth HIV-seropositive individuals provided 287 positives cultures from January 2000 to December 2006. There was positive correlation between tuberculosis and prison record $(p=0.021)$. Tobacco use reduced the mean lifetime from tuberculosis diagnosis to obit $(p=0.05)$. TCD4 ${ }^{+}$levels and a diffuse chest $X$-ray finding were associated to Mycobacterium tuberculosis (MT) isolation ( $p=0.014$ and 0.000 , respectively). Approximately eleven percent of all MT strains showed resistance to at least one drug while $3.1 \%$ were multidrug resistant. Non-tuberculous mycobacteria (NTM) totalized $35.19 \%$ of all mycobacteria and the most frequently isolated species were Mycobacterium avium complex (MAC; $22.3 \%$ ), M. fortuitum (5.2 \%) and $M$. gordonae $(3.1 \%)$. We conclude that the HIV-infected population studied remains with a high prevalence of NTM colonization in the post HAART era. In a wide country like Brazil, regional differences must be further evaluated in order to improve control and treatment of these opportunistic infections.

Key Words: Mycobacterium, HIV, Co-infection. 